\title{
Some Aspects of Non-Orthogonal Stagnation-Point Flow towards a Stretching Surface
}

\author{
Motahar Reza ${ }^{1}$, Anadi Sankar Gupta ${ }^{2}$ \\ ${ }^{1}$ Department of Mathematics, National Institute of Science \& Technology, Berhampur, India \\ ${ }^{2}$ Department of Mathematics, Indian Institute of Technology, Kharagpur, India \\ E-mail: reza@nist.edu \\ Received May 12, 2010; revised July 21, 2010; accepted August 3, 2010
}

\begin{abstract}
The problem of steady two-dimensional oblique stagnation-point flow of an incompressible viscous fluid towards a stretching surface is reexamined. Here the surface is stretched with a velocity proportional to the distance from a fixed point. Previous studies on this problem are reviewed and the errors in the boundary conditions at infinity are rectified. It is found that for a very small value of shear in the free stream, the flow has a boundary layer structure when $a / c>1$, where $a x$ and $c x$ are the free stream stagnation-point velocity and the stretching velocity of the sheet, respectively, $x$ being the distance along the surface from the stagnation-point. On the other hand, the flow has an inverted boundary layer structure when $a / c<1$. It is also observed that for given values of $a / c$ and free stream shear, the horizontal velocity at a point decreases with increase in the pressure gradient parameter.
\end{abstract}

Keywords: Oblique Stagnation-Point Flow, Stretching Surface

\section{Introduction}

The study of the flow of an incompressible viscous fluid over a stretching surface has important bearing on several technological and industrial processes. Problems such as the extrusion of polymers in melt-spinning, glass blowing, spinning of fibers a several metallurgical as well as metal-working processes involve certain aspects of flow over stretching sheets. Crane [1] obtained a similarity solution in closed analytical form for steady twodimensional flow of an incompressible viscous fluid caused solely by the stretching of an elastic sheet which moves in its own plane with a velocity varying linearly with distance from a fixed point.

Chiam [2] investigated steady two-dimensional orthogonal and oblique stagnation-point flow of an incompressible viscous fluid towards a stretching surface in the case when the parameter $b$ representing the ratio of the strain rate of the stagnation-point flow to that of the stretching surface is equal to unity. By removing this highly restrictive assumption $(b=1)$, Mahapatra and Gupta [3] analyzed the steady two-dimensional orthogonal stagnation-point flow of an incompressible viscous fluid to-wards a stretching surface in the general case $b \neq 1$. They observed that the structure of the boundary layer depends crucially on the value of $b$. Reza and Gupta [4] generalized the problem of an oblique stagnation-point flow over a stretching surface by Chiam [2] to include surface strain rate different from that of the stagnation flow. But since the displacement thickness arising out of the boundary layer on the surface was ignored in their boundary condition at infinity, the analysis in [4] is of doubt full validity. This was rectified in a paper by Lok, Amin and Pop [5]. However, these authors [5] did not take into account the pressure gradient parameter in the boundary condition at infinity. This is a serious omission since the pressure gradient parameter is linked to the free stream shear in the oblique stagnation-point flow (Drazin and Riley [6]). Hence the results of the paper in [5] are also of doubtful validity.

It is noted that planar oblique stagnation-point flow of an incompressible viscous flow of an incompressible viscous fluid towards affixed rigid surface was first studied by Stuart [7]. This problem was later independently investigated by Tamada [8] and Dorrepaal [9]. The analogue of the planar oblique stagnation-point flow to stagnation flow obliquely impinging on a rigid circular cylinder was discussed by Weidman and Putkaradze [10]. Exact similarity solutions for impingement of two viscous immiscible oblique stagnation flows forming a flat 
interface was given by Tilley and Weidman [11]. On the other hand heat transfer in oblique stagnation-point flow of an incompressible viscous fluid towards stretching surface was investigated by Mahapatra, Dholey and Gupta [12]. Further oblique stagnation-point flow of a viscoelastic fluid towards a stretching surface was studied by Mahapatra, Dholey and Gupta [13].

The objective of the present paper is to rectify the errors in [4] and [5] and give a correct solution to the above problem. It is worth pointing out that an oblique stagnation-point flow occurs when a separated viscous flow reattaches to a surface.

\section{Flow Analysis}

Consider the steady two-dimensional flow near a stagnation point when an incompressible viscous fluid impinges obliquely on an elastic surface coinciding with the plane $y=0$, the flow being confined to $y>0$. Two equal and opposite forces are applied along the $x$-axis so that the surface is stretched keeping the origin fixed, as shown in Figure 1. The velocity components in the inviscid free stream along the $x$ and $y$ directions are

$$
U_{0}=a x+2 b\left(y-\delta_{1}\right), \quad V_{0}=-a\left(y-\delta_{2}\right),
$$

respectively, where $a$ and $b$ are constants. Further $\delta_{2}$ is the displacement thickness arising out of the boundary layer on the stretching surface and $\delta_{1}$ is the parameter which controls the horizontal pressure gradient that produces the shear flow. Note that the whole flow field given by (1) may be viewed as being composed of an orthogonal stagnation-point flow combined with a horizontal shear flow. The corresponding stream function for the above velocity distribution is

$$
\psi_{0}=a x\left(y-\delta_{2}\right)+b\left(y-\delta_{1}\right)^{2}
$$

There appears a boundary layer on the surface at high Reynolds number. At the stretching surface, the no-slip condition gives

$$
u=c x, v=0 \text { at } y=0,
$$

where $c$ is a positive constant and $u$ and $v$ are the velocity components along $x$ and $y$ directions, respectively. In Reza \& Gupta [4], stream function in the boundary layer was assumed in the form

$$
\frac{\psi}{v}=\xi F(\eta)+W(\eta)
$$

where $v$ is the kinematic viscosity and

$$
\xi=x\left(\frac{c}{v}\right)^{\frac{1}{2}}, \eta=y\left(\frac{c}{v}\right)^{\frac{1}{2}},
$$

This gives the dimensionless velocity components from (4) and (5) as

$$
U=\xi F^{\prime}(\eta)+W^{\prime}(\eta), V=-F(\eta),
$$

where $U=u /(c v)^{1 / 2}$ and $V=v /(c v)^{1 / 2}$. Using (6) in the Navier-Stokes equations it was shown in [4] that $F(\eta)$ and $W(\eta)$ satisfy the following equations

$$
\begin{aligned}
& F^{\prime 2}-F F^{\prime \prime}-F^{\prime \prime \prime}=c_{1}, \\
& F^{\prime} W^{\prime}-F W^{\prime \prime}-W^{\prime \prime \prime}=c_{2},
\end{aligned}
$$

where $c_{1}$ and $c_{2}$ are constants. From (6), no-slip conditions (3) become

$$
\begin{aligned}
& F(0)=0, F^{\prime}(0)=1, \\
& W(0)=0, W^{\prime}(0)=0 .
\end{aligned}
$$

Further from (1) and (6), the boundary condition for $F(\eta)$ and $W(\eta)$ at infinity are

$$
\begin{gathered}
F^{\prime}(\eta) \sim \frac{a}{c} ; F(\eta) \sim \frac{a}{c}\left(\eta-d_{2}\right) \text { as } \eta \rightarrow \infty, \\
W^{\prime}(\eta) \sim 2 \frac{b}{c}\left(\eta-d_{1}\right) \text { as } \eta \rightarrow \infty,
\end{gathered}
$$

where $d_{2}\left(=(c / v)^{\frac{1}{2}} \delta_{2}\right)$ is the dimensionless displacement thickness parameter and $d_{1}\left(=(c / v)^{\frac{1}{2}} \delta_{1}\right)$ is the dimensionless pressure gradient parameter linked to the free stream shear flow.

Reza and Gupta [4] ignored both the constants $\delta_{1}$ and $\delta_{2}$ in (1). While pointing out that $\delta_{2}$ should be taken into account (as mentioned in the Introduction), Lok, Amin \& Pop [5] rectified this error in [4]. However, these authors in [5] lost sight of the constant $\delta_{1}$ in (1) and consequently arrived at governing equations for the velocity distribution one of which is incorrect. Hence their analysis is of doubtful validity.

Using the boundary conditions (11) and (12) in (7) and (8), we get

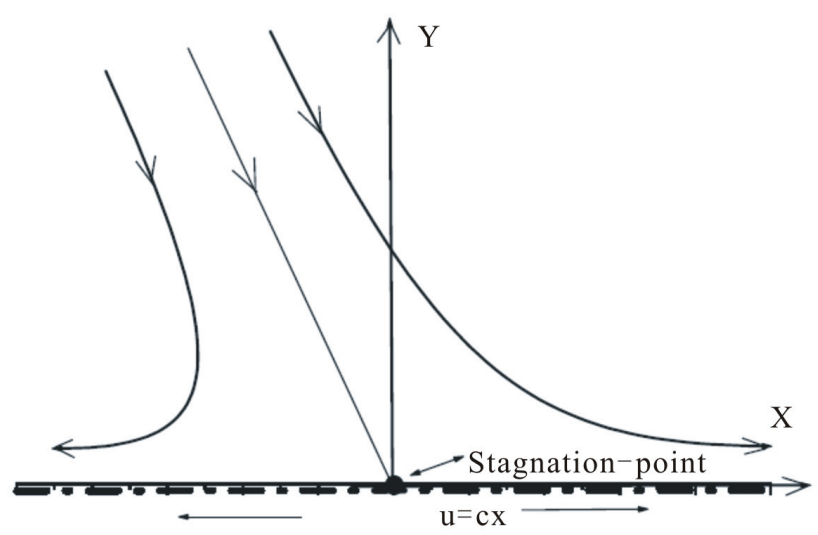

Figure 1. A sketch of the physical problem. 


$$
c_{1}=\frac{a^{2}}{c^{2}}, c_{2}=2 \frac{a b}{c^{2}}\left(d_{2}-d_{1}\right) .
$$

Thus the governing equations for $F(\eta)$ and $W(\eta)$ become

$$
\begin{gathered}
F^{\prime 2}-F F^{\prime \prime}-F^{\prime \prime \prime}=\frac{a^{2}}{c^{2}}, \\
F^{\prime} W^{\prime}-F W^{\prime \prime}-W^{\prime \prime \prime}=2 \frac{a b}{c^{2}}\left(d_{2}-d_{1}\right) .
\end{gathered}
$$

Note that Equation (15) derived by Lok et al. [5] does not include $d_{1}$. Further the boundary condition (12) in [5] is also erroneous due to the absence of $d_{1}$. Substitution of (14) and (15) in the $x$ and $y$ momentum equations followed by integration gives the pressure distribution $p(x, y)$ in the flow as

$$
\begin{aligned}
& \frac{p(x, y)}{c \rho v} \\
& =-\frac{1}{2}\left(\frac{a^{2}}{c^{2}} \xi^{2}+F^{2}\right)-F^{\prime}-2 \frac{a b}{c^{2}}\left(d_{2}-d_{1}\right) \xi+\text { constant. }
\end{aligned}
$$

which can be found once $F(\eta)$ is known.

Equations (14) and (15) subject to the boundary conditions (9)-(12) are solved numerically by finite difference method using Thomas algorithm (Fletcher [14]).

\section{Results and Discussion}

Figure 2 shows the variation of $U(\xi, \eta)$ with $\eta$ at a fixed value of $\xi(=0.5)$ for several values of $a / c$ when the pressure gradient parameter $d_{1}=0.5$ and $b / c$ $=1.0$. It can be seen that at a given value of $\eta, U$ increases with increase in $a / c$. Further when $b / c$ is very small and equal to 0.05 , say, the velocity profile at a fixed value of $\xi(=0.5)$ for several values of $a / c$ with $d_{1}=0.5$ shows a boundary layer structure (see Figure 3) and the thickness of the boundary layer decreases with increase in $a / c$. From a physical point of view, this stems from the fact that increase of straining motion in the free stream (e.g., increase in $a / c$ for a fixed value of $c$ ) leads to increase in acceleration of the free stream. This results in thinning of boundary layer.

Figure 3 shows that when the free stream shear is negligible ( $b / c=0.05$ for a given value of $c$ ), the flow has a boundary layer structure because in this case straining motion dominates over the shear. However, this boundary layer structure is affected to a great extent in the presence of considerable shear in the free stream (see Figure 2).

The dimensionless displacement thickness $d_{2}$ is computed for different values of $a / c$ from the solution of Equation (14) subject to the boundary conditions (9) and (11) and shown in the above Table 1. It may be noticed that for $a / c=1$, displacement thickness is approximately zero (numerically). This is due to fact that when $a / c=1$, the stretching velocity of the plate is precisely equal to the irrotational straining velocity. From a physical point of view, the absence of boundary layer in this case arises from the fact that although the flow is not frictionless in a strict sense, the friction is uniformly distributed and does not therefore affect the motion. Stuart [7] and Tamada [8] showed that the value of the dimensional displacement thickness is 0.6479 for oblique stagnation point flow over a rigid plate. This result can be compared with that of our problem by considering $c=0$ in the boundary condition (3) which gives $F(0)=0$ and $F^{\prime}(0)=0$. We have found that the value of the displacement thickness is $d_{2}=0.64788$. It may be noted that in both the studies of Stuart [7] and Tamada [8], the pressure gradient parameter $\delta_{1} \neq 0$.

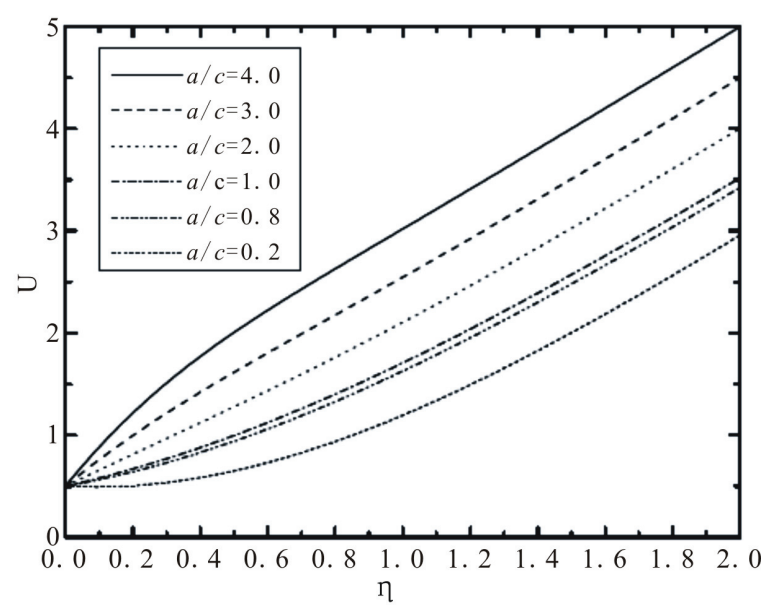

Figure 2. Variation of $U(\xi, \eta)$ with $\eta$ at $\xi=0.5$ for several values of $a / c$ when $d_{1}=0.5$ and $b / c=\mathbf{1 . 0}$.

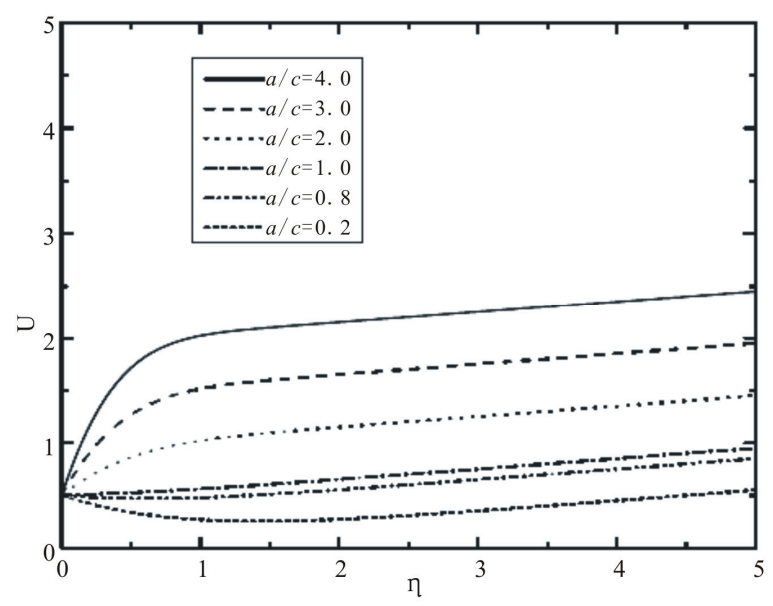

Figure 3. Variation of $U(\xi, \eta)$ with $\eta$ at $\xi=0.5$ for several values of $a / c$ when $d_{1}=0.5$ and $b / c=\mathbf{0 . 0 5}$. 
Table 1. Values of the displacement thickness $d_{2}$ for several values of $a / c$.

\begin{tabular}{ccccc}
\hline$a / c$ & 3 & 2 & 1.5 & 1.0 \\
\hline$d_{2}$ & 0.235278 & 0.2082290 & 0.1548889 & $9.3602152 \times 10^{-7}$ \\
\hline
\end{tabular}

Figure 4 displays the variation of $U(\xi, \eta)$ with $\eta$ at a fixed location $\xi(=0.5)$ for several values of the pressure gradient parameter $d_{1}$ when $a / c=3$ and $b / c$ $=1.0$. It may be seen that at a given value of $\eta$, the horizontal velocity $U$ decreases with increase in $d_{1}$.

The streamline patterns for the oblique stagnationpoint flow are shown in Figures 5(a) and 5(b) for very small value of the free stream shear $b / c=0.05$ and $d_{1}=0.4$ in two cases 1) $\left.a / c=0.2,2\right) a / c=5.0$. It can be seen that for $a / c<1$, the stream lines are slightly tilted towards the left. but when $a / c$ is large (= 5 ), the flow almost resembles that of an orthogonal stagnation-point flow as long as the free stream shear is very small (see Figure 5(b)). For moderate value of free stream shear $(b / c=1)$, the disposition of the streamlines is shown in Figures 6(a) and 6(b) for $a / c=0.2$, and $a / c=2.0$, respectively. It is observed from Figures 5(a) and 6(a) that for a given value of $a / c(=0.2)$, with increase in the free stream shear, the streamlines become more tilted towards the left. We also find that with increase in the straining motion in the free stream, the streamlines are less and less tilted to the left. This is plausible on physical grounds because with increase in $a / c$ for a given value of $b / c$, the flow tends to resemble an orthogonal stagnation-point flow.

\section{Summary}

An exact solution of the Navier-Stokes equations is given which represents steady two-dimensional oblique

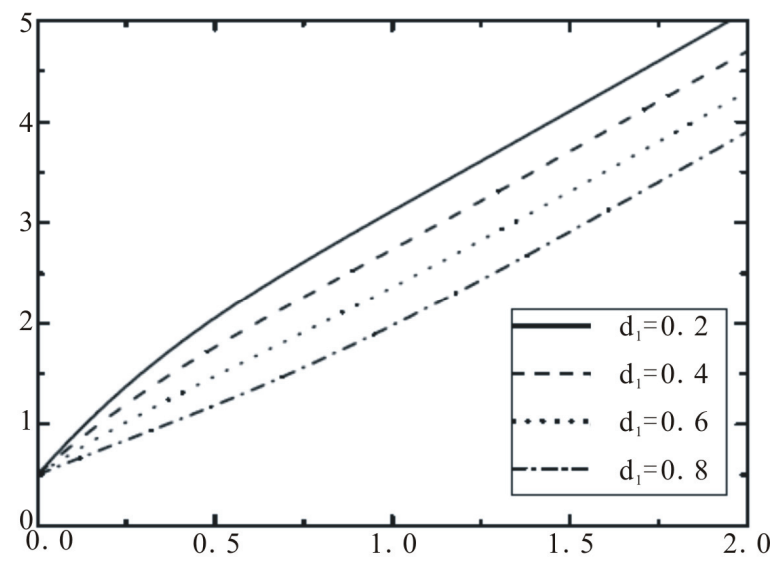

Figure 4. Variation of $U(\xi, \eta)$ with $\eta$ at $\xi=0.5$ for several values $d_{1}$ when $a / c=3.0$ and $b / c=\mathbf{1 . 0}$.

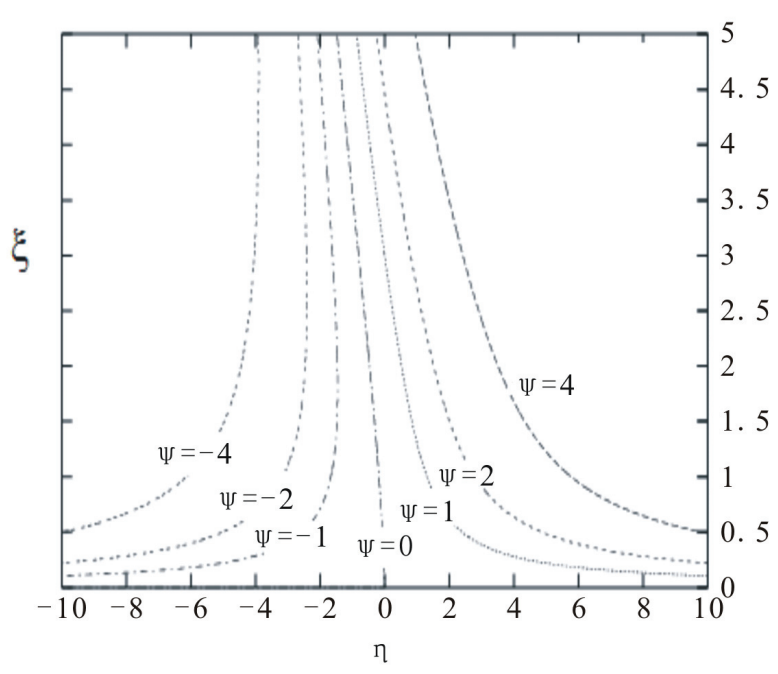

(a) when $a / c=0.2$

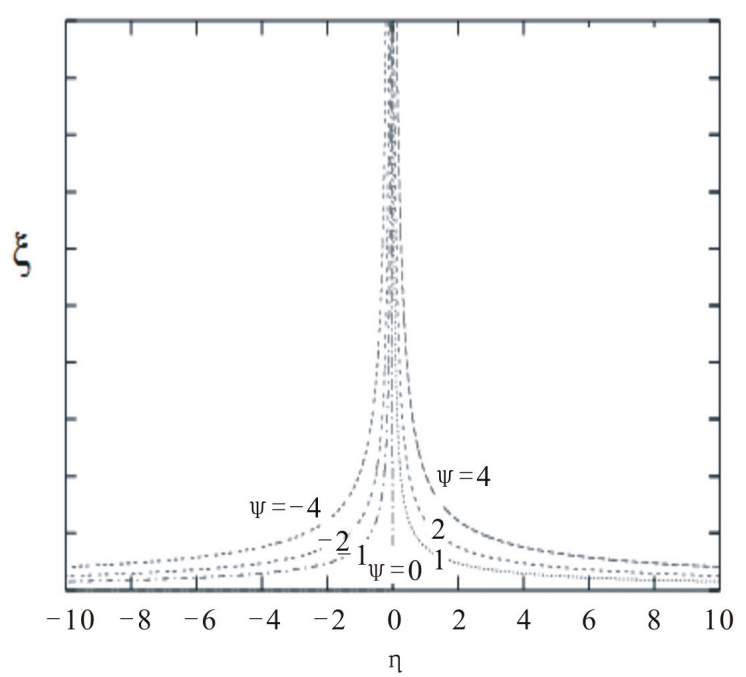

(b) when $a / c=5.0$

Figure 5. Streamline patterns for $b / c=\mathbf{0 . 0 5}$ and $d_{\mathbf{1}}=\mathbf{0 . 4}$ (a) when $a / c=\mathbf{0 . 2}$ (b) when $a / c=\mathbf{5 . 0}$.

stagnation-point flow of an incompressible viscous fluid towards a surface stretched with velocity proportional to the distance from a fixed point. It is shown that when the free stream shear is negligible, the flow has a boundary layer behaviour when the stretching velocity is less than the free stream velocity $(a / c>1)$, and it has an inverted boundary layer structure when just the reverse is true $(a / c<1)$. It is found that the obliquity of the flow towards the surface increases with increase in $b / c$. This is consistent with the fact that increase in $b / c$ (for a fixed value of $a / c$ ) results in increase in the shearing motion which in turn leads to increased obliquity of the flow towards the surface. 


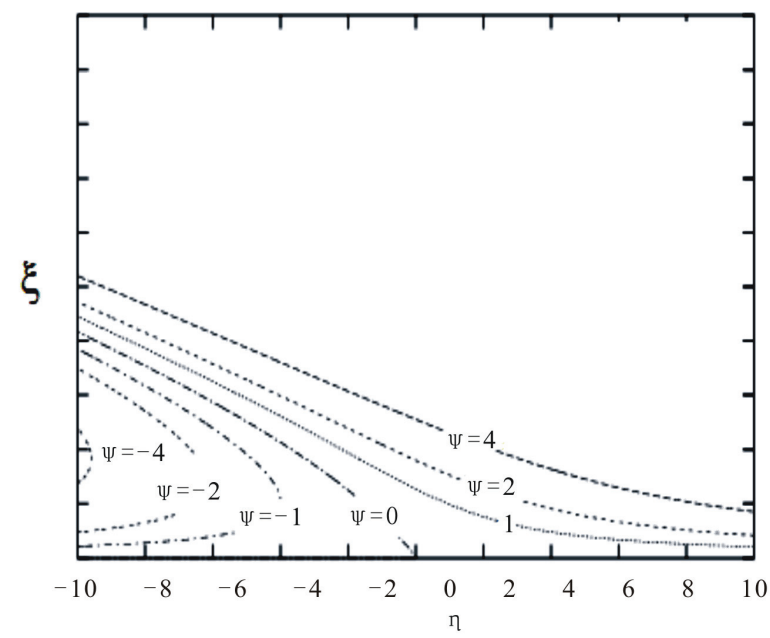

(a) when $a / c=0.2$.

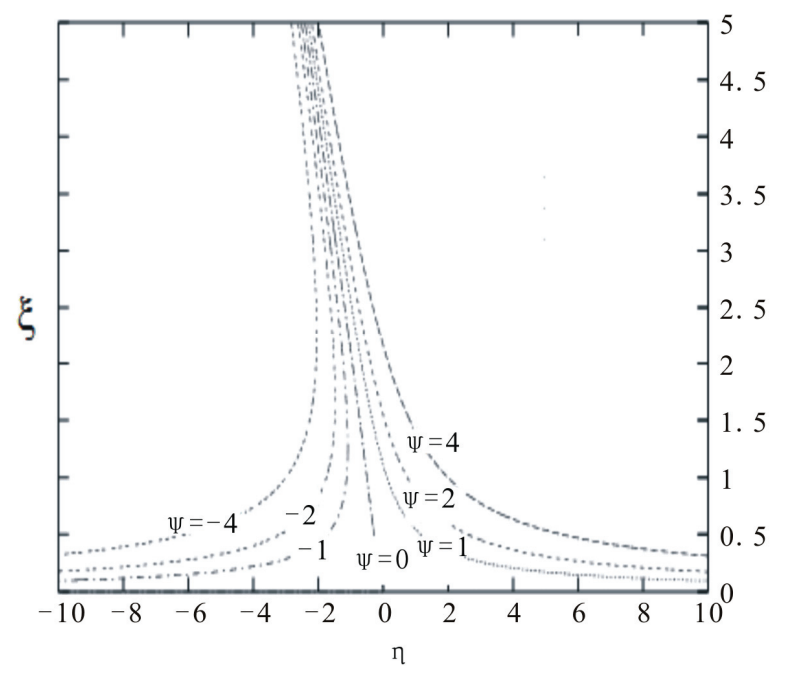

(b) when $a / c=2.0$

Figure 6. Streamline patterns for $b / c=1.0$ and $d_{1}=0.4$ (a) when $a / c=\mathbf{0 . 2}$; (b) when $a / c=\mathbf{2 . 0}$.

\section{Acknowledgements}

One of the authors (A. S. G) acknowledges the financial assistance of Indian National Science Academy, New Delhi for carrying out this work. Authors would also like to acknowledge the use of the facilities and technical assistance of the Center of Theoretical Studies at Indian Institute of Technology, Kharagpur.

\section{References}

[1] L. J. Crane, "Flow Past a Stretching Plate," Zeitschrift für angewandte Mathematik und Physik, Vol. 21, 1970, pp. 645-657.

[2] T. C. Chiam, "Stagnation-Point Flow towards a Stretching Plate," Journal of Physical Society of Japan, Vol. 63, No. 6, 1994, pp. 2443-2444.

[3] T. R. Mahapatra and A. S. Gupta, "Heat Transfer in Stagnation-Point Flow towards a Stretching Sheet," Heat and Mass Transfer, Vol. 38, No. 6, 2002, pp. 517-521.

[4] M. Reza and A. S. Gupta, "Steady Two-Dimensional Oblique Stagnation Point Flow towards a Stretching Surface," Fluid Dynamics Research, Vol. 37, No. 5, 2005, pp. 334-340.

[5] Y. Y. Lok, N. Amin and I. Pop, "Non-Orthogonal Stagnation Point towards a Stretching Sheet," International Journal of Non-Linear Mechanics, Vol. 41, No. 4, 2006, pp. 622-627.

[6] P. G. Drazin and N. Riley, "The Navier-Stokes Equations: A Classification of Flows and Exact Solutions," Cambridge University Press, Cambridge, 2006.

[7] J. T. Stuart, "The Viscous Flow near a Stagnation Point when External Flow has Uniform Vorticity," Journal of the Aero/Space Sciences, Vol. 26, 1959, pp. 124-125.

[8] K. Tamada, "Two-Dimensional Stagnation-Point Flow Impinging Obliquely on a Plane Wall," Journal of Physical Society of Japan, Vol. 46, No. 1, 1979, pp. 310-311.

[9] J. M. Dorrepaal, “An Exact Solution of the Navier-Stokes Equation which Describes Non-Orthogonal StagnationPoint Flow in Two Dimensions," Journal of Fluid Mechanics, Vol. 163, 1986, pp. 141-147.

[10] D. Weidman and V. Putkaradzeb, “Axisymmetric Stagnation Flow Obliquely Impinging on a Circular Cylinder," European Journal of Mechanics - B/Fluids, Vol. 22, No. 2, 2003, pp. 123-131.

[11] B. S. Tilley, P. D. Weidman, "Oblique Two-Fluid Stagnation-Point Flow," European Journal of Mechanics B/Fluids, Vol. 17, No. 2, 1998, pp. 205-217.

[12] T. R. Mahapatra, S. Dholey and A. S. Gupta, "Heat Transfer in Oblique Stagnation-Point Flow of an Incompressible Viscous Fluid towards a Stretching Surface," Heat and Mass Transfer, Vol. 43, No. 8, 2007, pp. 767-773.

[13] T. R. Mahapatra, S. Dholey and A. S. Gupta, "Oblique Stagnation-Point flow of an Incompressible Visco-Elastic Fluid towards a Stretching Surface," International Journal of Non-Linear Mechanics, Vol. 42, No. 3, 2007, pp. 484-499.

[14] C. A. J. Fletcher, "Computational Techniques for Fluid Dynamics,” Vol. 2, Springer-Verlag, Berlin, 1988. 